INPLASY

PROTOCOL

To cite: Liu et al. Efficacy and safety of Whole-lung lavage for pulmonary alveolar proteinosis:

A protocol for systematic review and meta-analysis. Inplasy protocol 202180111. doi:

10.37766/inplasy2021.8.0111

Received: 29 August 2021

Published: 29 August 2021

Corresponding author:

Shixu Liu

loliduc@163.com

Author Affiliation:

Guang'anmen Hospital, China

Academy of Chinese Medical

Sciences.

Support: NSFC.

Review Stage at time of this submission: Piloting of the study selection process.

\section{Efficacy and safety of Whole-lung lavage for pulmonary alveolar proteinosis: A protocol for systematic review and meta-analysis}

Liu, SXㅍ Xia, K²; Xiong, MR³; Duan, YY4; Cui, XN5; Li, GX6.

Review question / Objective: To investigate the therapeutic effect and safety of whole-lung lavage (WLL) for pulmonary alveolar proteinosis (PAP). P: Patients with pulmonary alveolar proteinosis; I: Treatments with whole-lung lavage; C: Treatments without whole-lung lavage; 0 : Curative effects, adverse effects; S: Observational studies.

Information sources: PubMed, Cochrane Library, EMBASE, CNKI, Wanfang, VIP, and CBM databases will be searched for the identification of eligible data (last updated search in September, 2021). Additionally, potential studies in the reference lists of valid studies will also be considered as the information source for supplement.

INPLASY registration number: This protocol was registered with the International Platform of Registered Systematic Review and Meta-Analysis Protocols (INPLASY) on 29 August 2021 and was last updated on 29 August 2021 (registration number INPLASY202180111).

Conflicts of interest:

None declared.

\section{INTRODUCTION}

Review question / Objective: To investigate the therapeutic effect and safety of wholelung lavage (WLL) for pulmonary alveolar proteinosis (PAP). P: Patients with pulmonary alveolar proteinosis; I: Treatments with whole-lung lavage; C: Treatments without whole-lung lavage; 0 :
Curative effects, adverse effects; S: Observational studies.

Condition being studied: Pulmonary alveolar proteinosis (PAP), first described by Rosen et al. in 1958, is a rare pulmonary surfactant homeostasis disorder resulting in buildup of lipo-proteinaceous material within the alveoli, whose annual prevalence 
was estimated to be 3.7-6.2 per million. The accumulation of lipoproteinaceous-rich materials was caused by the disability of macrophages to clear alveolar surfactants, which could result in restrictive pulmonary ventilation dysfunction, decreased diffusion capacity, and even could progress to respiratory failure. The current standard treatment in most types of PAP is wholelung lavage(WLL). Although it is widely considered by practitioners to improve symptoms, radiological abnormalities and oxygenation in patients, there has not been any systematic evaluation of the clinical efficacy and safety of WLL. In severe cases of PAP, WLL was even considered potentially harmful for the poor outcomes. Therefore, aiming to assess the quantity, quality and overall strength of existing clinical evidence on WLL in the treatment of PAP, we attempt to perform a systematic review and meta-analysis to identify whether the application of WLL appeared to be adequate reliability regarding on the efficacy and safety.

\section{METHODS}

Participant or population: Patients with pulmonary alveolar proteinosis (PAP).

Intervention: Treated with whole-lung lavage (WLL).

Comparator: Treated without whole-lung lavage (WLL).

Study designs to be included: Both controlled and uncontrolled studies.

Eligibility criteria: Inclusion criteria:(1) The controlled and uncontrolled studies using WLL in PAP patients aged 18 years or older; (2) The PAP patients were diagnosed by open lung biopsy or transbronchial lung biopsy; (3) Included studies supplied complete data including number of pathological types, arterial partialpressure of oxygen (PaO2), carbon monoxide diffusion capacity (DLCO), the forced expiratory volumein 1 second (FEV1), forced vital capacity (FVC), and so on; (4) After careful reexamination, only the latest complete study was included when the extracted studies were published by the same authors.Exclusion criteria:(1)Studies in which the clinical outcome was not reported; (2)Single-patient case report or short case series involving fewer than 3 patients; (3) Participants were less than 18year old. (4) Multiple publications from the same study(1)The controlled and uncontrolled studies using WLL in PAP patients aged 18 years or older; (2)The PAP patients were diagnosed by open lung biopsy or transbronchial lung biopsy; (3)Included studies supplied complete data including number of pathological types, arterial partialpressure of oxygen (PaO2), carbon monoxide diffu-sion capacity (DLCO), the forced expiratory volumein 1 second (FEV1), forced vital capacity (FVC), and so on; (4)After careful reexamination, only the latest complete study was included when the extracted studies were published by the same authors.

Information sources: PubMed, Cochrane Library, EMBASE, CNKI, Wanfang, VIP, and CBM databases will be searched for the identification of eligible data (last updated search in September, 2021). Additionally, potential studies in the reference lists of valid studies will also be considered as the information source for supplement.

Main outcome(s): Response rates, relapse rates, blood gas analysis $(\mathrm{PaO} 2, \mathrm{PaCO}$, and SaO2), and lung function (DLCO, FEV1, and FVC).

Quality assessment / Risk of bias analysis: The quality of all included studies will be assessed using the Agency for Healthcare Review and Quality (AHRQ) criteria for observational studies. Each study was assessed by two investigators independently.

Strategy of data synthesis: The RevMan 5.3 software will be used for meta-analysis. The relative risk(RR) will be used as the effect index for the dichotomous variable, standardized mean differences (SMD) will be used as the effect index for the continuous variable. The confidence interval (Cl) of each effect index was set to $95 \%$. 12 statistic will be adopted to assess 
the heterogeneity. If there is heterogeneity between the studies (12>50\%), the random effects model will be selected; Otherwise, the fixed effect model will be utilized. $P<0.05$ indicates statistical significance. In addition, if the clinical data provided by the included studies are incomplete and cannot be systematically evaluated, the descriptive analysis shall be carried out.

Subgroup analysis: When there is obvious heterogeneity in the study, subgroup analysis will be conducted according to the proportions of GM-CSF therapy, age of patients, proportions of men and proportions of smokers.

Sensitivity analysis: We will use the sensitivity analysis to assess the stability of the findings. Low-quality literature can be excluded for sensitivity analysis.

Country(ies) involved: China.

Keywords: Whole-lung lavage; pulmonary alveolar proteinosis; meta-analysis; protocol.

Contributions of each author:

Author 1 - Shixu Liu.

Email: loliduc@163.com

Author 2 - Kun Xia.

Email: 361347735@qq.com

Author 3 - Mengran Xiong.

Email: grandmeeting@163.com

Author 4 - Yuanyuan Duan.

Email: 1259439743@qq.com

Author 5 - Xiangning Cui.

Email: cuixiangning@126.com

Author 6 - Guangxi Li.

Email: Igx0410@163.com 